Nervenarzt 2019 · 90:307

https://doi.org/10.1007/s00115-019-0684-8

Online publiziert: 26. Februar 2019

(c) Springer Medizin Verlag $\mathrm{GmbH}$, ein Teil von Springer Nature 2019

CrossMark

Johannes Noth

Neurologische Klinik, Universitätsklinikum der RWTH Aachen, Aachen, Deutschland

\title{
Verwischte Grenze zwischen evidenzbasierter Medizin und therapeutischen Verfahren
}

satz bei unseren Patienten prüfen. Und so scheint die herkömmliche und oft auch ideologisch gemünzte Konfrontation von ,Schulmedizin" und ,Alternativmedizin" eher sinnlos zu sein." Im letzten Punkt hat der Autor Recht. Der Ausdruck Schulmedizin ist tatsächlich sinnlos und sollte zugunsten des Begriffs ,,evidenzbasierte Medizin“ fallen gelassen und damit gegen die „nichtevidenzbasierte Medizin“ abgegrenzt werden. Jedem Patienten sollte es überlassen bleiben, die Therapieform zu wählen, die seinem Naturell entspricht - nur kann unser Sozialsystem nicht für die zahlreichen „alternativen“ Therapieangebote aufkommen.

Ich möchte drei Beispiele für die Widersprüchlichkeit des von Herrn Prof. Juckel vorgestellten Konzepts nennen:

1. Wie können Universitäten unseren Medizinstudenten eine naturwissenschaftlich begründete Medizin vermitteln wollen, wenn sie andererseits Verfahren, wie die Homöopathie, die sich jeder naturwissenschaftlichen Erklärung entziehen, in die Lehrpläne aufnehmen. Die Homöopathie hat ohne Zweifel für viele, die von dieser Methode überzeugt sind, einen starken suggestiven Einfluss. Sie sollte, da sie nicht evidenzbasiert ist, jedoch nicht von den Krankenkassen finanziert werde. Das Gleiche gilt für die Akupunktur und viele andere nicht evidenzbasierte Therapieverfahren.

2. Wenn "gut gesicherte Aspekte in das medizinische Repertoire integriert werden sollten" - was immer dieser Satz bedeuten mag -, dann verschiebt sich damit nur die Grenze $\mathrm{zu}$ anderen, noch exotischeren, Behandlungsverfahren, die dann auch hoffähig werden könnten. Mit welchen Argumenten könnten diese Verfahren dann abgewehrt werden, wenn der einzige Maßstab, der Evidenznachweis, nicht zählt. Der Weg zur Anerkennung der von Herrn Prof. Juckel selbst genannten, zum Teil recht dubiosen Verfahren wie „Geistheilen“, ist dann nicht mehr weit.

3. Warum sollten alternative Verfahren immer nur komplementär wirken und nicht für sich allein? Am ehesten wohl deshalb, weil bei einem nichtkomplementären Einsatz die fehlende Wirksamkeit - über den Placeboeffekt hinaus - viel einfacher nachgewiesen werden könnte.

Deshalb zum Schluss meine Warnung frei nach Goethe: „Herr, die Not ist groß. Die ich rief, die Geister, werd ich nun nicht los." Hoffentlich gilt dies nicht für den in diesem Leitthema eingeschlagenen Weg in die alternative Medizin.

\section{Korrespondenzadresse}

Prof. Dr. Johannes Noth

Neurologische Klinik, Universitätsklinikum der RWTH Aachen

Pauwelsstraße 30, 52074 Aachen, Deutschland jnoth@ukaachen.de

Interessenkonflikt. J. Noth gibt an, dass kein Interessenkonflikt besteht. 